\title{
Revisión
}

\section{La comunicación en la relación médico-paciente. Análisis de la literatura científica entre 1990 y 2010}

\author{
Pedro Cófreces \\ Silvia Deborah Ofman \\ Dorina Stefani \\ Instituto de Investigaciones Cardiológicas (ININCA - UBA) \\ Consejo Nacional de Investigaciones Científicas y Técnicas (CONICET) \\ (Buenos Aires, Argentina)
}

\section{Palabras clave}

comunicación médico-paciente relación médico-paciente satisfacción del paciente adherencia al tratamiento

\begin{abstract}
Resumen
El objetivo del trabajo es presentar los principales hallazgos de estudios teóricos y empíricos sobre la comunicación en la relación médico-paciente y su impacto en la satisfacción y adherencia del paciente al tratamiento médico, a partir de la literatura científica publicada entre 1990 y 2010 en la sociedad occidental. Se realizó la búsqueda del material bibliográfico en bases de datos y bibliotecas, durante el periodo febrero - noviembre de 2012. Se consultaron 143 artículos en total y fueron seleccionados 63. Los criterios de inclusión han sido en función de los ejes temáticos emergentes del análisis de contenido previo y de acuerdo al objetivo planteado. Fueron excluidos aquellos con un tratamiento no riguroso sobre el tema y sin un enfoque relacional. La integración de toda la información sobre el tema, que en otras publicaciones aparecía por separado, es el principal aporte de esta revisión, cuyas principales conclusiones fueron: 1) en la relación médico-paciente se destaca la correlación entre la calidad del proceso comunicativo, la satisfacción del usuario y el cumplimiento de los tratamientos; 2) en la interacción médico - paciente, la satisfacción del enfermo y la adherencia al tratamiento son fenómenos multidimensionales, en los que inciden múltiples cuestiones; 3) se halló un mayor riesgo de abandono al tratamiento en sistemas de salud con una atención centralizada, alta demanda, poco personal, demoras y atención menos personalizada; y 4) altos niveles de adherencia se asociaron con modelos de comunicación que consideran las actitudes, creencias, preferencias de los pacientes y permiten un proceso de negociación con mayor acuerdo y comprensión.
\end{abstract}




\section{Communication within the doctor - patient relationship. Analysis of scientific literature between 1990 and 2010.}

\begin{tabular}{c}
\hline Keywords \\
\hline doctor-patient communication \\
doctor-patient relationship \\
patient satisfaction \\
treatment adherence
\end{tabular}

\section{Cómo citar el artículo}

Cófreces, P.; Ofman, S. y Stefani, D. (2014). La comunicación en la relación médico-paciente. Análisis de la literatura científica entre 1990 y 2010. Revista de Comunicación y Salud, Vol. 4, pp. 19-34.

DOI: http://doi.org/10.35669/revistadecomunicacionysalud.2014.4.19-34 


\section{Introducción}

Si bien la comunicación médico-paciente es tan antigua como la medicina misma, no fue hasta época reciente, fines de la década del 70 del siglo XX, que ha sido abordada de modo sistemático y formal. La investigación en comunicación y salud es reciente, con desarrollo en los años 80 y fundamentalmente en la década del 90 (Bellón y Martínez, 2001). La profundización de su estudio coincide con el enfoque de considerar importante la relación centrada en el paciente, que se inicia en esos años, en correspondencia con los movimientos sociales que defendían los derechos y la autonomía del paciente (Laine y Davidoff, 1996; Vidal y Benito, 2010).

Dicha relación se ha transformado fundamentalmente a fines del siglo XX con la posmodernidad, a partir del capitalismo globalizado. Antes la relación era paternalista, donde el médico tenía un rol activo y el paciente acataba pasivamente sus indicaciones. El médico de familia centralizaba toda la información, lo que no sucede en la actualidad, producto de las especializaciones médicas donde se produce una fragmentación de dicha información. Las habilidades diagnósticas clásicas de observación, palpación, percusión, auscultación se fueron perdiendo, producto del desarrollo tecnológico que dio lugar al modelo tecno-médico. Esta nueva etapa histórica modificó la medicina, la practica médica y también generó cambios en las expectativas de los pacientes, así como cambios sociales (Dörr Anneliese, 2004; Pérgola, 2002; Pérgola, 2010). Estas transformaciones influyen en la relación médico-paciente a tal punto que en la actualidad se plantea que la misma atraviesa una crisis (Piñeiro, 2005; Pérgola, 2010).

Por su parte, la comunicación eficaz médico-paciente se ha relacionado positivamente con la calidad de la atención, la satisfacción de los pacientes y médicos, y los resultados de salud (Bellón y Martínez, 2001; Robbins et al., 1993). Asimismo, se ha probado la influencia de la calidad de la relación médico - paciente en la adherencia del paciente al tratamiento médico prescripto (McLane et al., 1995). Otra variable que incide en la relación médico - paciente es la referida a las competencias comunicacionales de los pacientes, que han sido muy poco estudiadas, y fundamentalmente la incidencia de los factores contextuales donde se desarrolla la entrevista clínica.

A partir de estas consideraciones, el objetivo del trabajo es presentar un análisis de los principales hallazgos obtenidos entre 1990 y 2010 a través de estudios teóricos y empíricos sobre la comunicación en la relación médico-paciente y su impacto en la satisfacción y adherencia del paciente al tratamiento médico prescripto.

\section{Método}

El método utilizado fue, de acuerdo a la pertinencia del objetivo, la consulta del material bibliográfico generado entre los años 1990 y 2010 en América Latina, Estados Unidos y Europa, tomando como palabras claves en español: "comunicación médicopaciente", "relación médico-paciente", "entrevista clínica", "satisfacción de pacientes" y "adherencia al tratamiento". La búsqueda se realizó durante el periodo febrero - noviembre de 2012. Se consultaron 143 artículos y fueron seleccionados 63. Los criterios de inclusión de los artículos han sido en función de los ejes temáticos emergentes 
del análisis de contenido previo y de acuerdo al objetivo planteado. Fueron excluidos los artículos con un tratamiento no riguroso sobre el tema y que a la vez no tenían un enfoque relacional sobre la cuestión. La integración de toda la información sobre el tema, que en otras publicaciones aparecía por separado, es el principal aporte de esta revisión.

Las bases de datos electrónicas utilizadas fueron: Redalyc (Red de Revistas Científicas de América Latina y el Caribe, España y Portugal), Dialnet (Universidad de la Rioja, España), MEDLINE (National Library of Medicine), Oxford Journals (Oxford University Press), CLACSO (Consejo Latinoamericano de Ciencias Sociales) y Pubmed (US National Library of Medicine). Dicha búsqueda electrónica fue completada a partir de las listas de referencias de los trabajos elegidos con una búsqueda manual en bibliotecas de instituciones educativas y públicas, tales como las facultades de Medicina, Ciencias Sociales y Psicología de la Universidad de Buenos Aires.

\section{Resultados}

Con la finalidad de poder explorar y analizar cada uno de los tópicos que intervienen en el objetivo planteado, el material bibliográfico seleccionado y evaluado en forma cualitativa fue sistematizado en cuatro ejes temáticos: modelos de relación médico paciente; habilidades comunicacionales del médico en la entrevista clínica; satisfacción del paciente; y adherencia al tratamiento médico.

\subsection{Modelos de Relación Médico - Paciente}

a) Definición

Pérez Cicili et al. la definen así:

"Es una relación interpersonal de tipo profesional que sirve de base a la gestión de salud. Se trata de una relación donde se presta un servicio de alta significación, por ser la salud uno de las más preciadas aspiraciones del ser humano, y a diferencia de las relaciones interpersonales condicionales, la situación profesional más frecuente es la entrega total, sin aspirar a reciprocidad alguna en los aspectos señalados (...) La práctica médica, desde sus orígenes ha estado estrechamente vinculada a la comunicación interpersonal y al desarrollo de las habilidades clínicas. Dado su carácter de relación social la relación médico-paciente lleva la impronta del contexto social donde se desarrolla" (2003).

De hecho, la relación médico - paciente se ha considerado como uno de los factores de mayor influencia en el cumplimiento de las recomendaciones terapéuticas (Di Matteo et al., 1993; Froján y Rubio, 2005). Asimismo, la importancia del proceso de comunicación en dicha interacción ha sido estudiada por varios autores, que apuntan a la correlación existente entre la calidad del proceso comunicativo, la satisfacción del usuario y el cumplimiento de los tratamientos (Ley, 1983; Lassen, 1991; Barca et al., 2004). 
b) Modelos de relación médico - paciente y grado de efectividad

Rodríguez Silva (2006) plantea que existen distintos modelos de relación médico- paciente, pero la más utilizada por su sentido práctico es aquella que establece tres formas distintas:

- Relación activo-pasiva (modelo paternalista): se establece, por lo general, con enfermos en estado de coma, o que se encuentran en una situación que no les permite establecer una relación más participativa.

- Relación de cooperación guiada (modelo autonomista donde decide el paciente y el médico tiene el rol de consultor): se establece con pacientes que están en condiciones de cooperar en su diagnóstico y tratamiento, como ocurre en algunas enfermedades agudas y crónicas.

- Relación de participación mutua (modelo de toma de decisiones compartidas): no sólo contempla el cumplimiento del tratamiento. También el control en discusión de situaciones, y las actitudes relacionadas con la causa y evolución de la enfermedad.

Por su parte, Hernández Torres y colaboradores (2006) describen cuatro modelos de comunicación en la relación médico - paciente, que implican estilos de comunicación entre ambos actores sociales:

- Modelo de las tres funciones del médico: consiste en recolectar información, responder a las emociones del paciente y educar e influenciar la conducta del mismo para su mejor manejo.

- Modelo clínico centrado en el paciente: presupone que la experiencia del enfermo se mueve cada vez a niveles más abstractos, que se necesita comprender el significado de la enfermedad para el paciente y sugiere también una comprensión compartida del médico y paciente acerca de los fundamentos comunes del problema y su manejo.

- Modelo de abordaje sistémico familiar para el cuidado del paciente: desarrollado desde la óptica de la terapia familiar y la teoría general de sistemas. La familia es considerada el contexto más relevante que puede influir en la salud, la enfermedad, y resulta clave para la obtención de buenos resultados.

- Modelo del autoconocimiento del médico: parte del supuesto de que a partir del conocimiento que el médico obtenga de sus propios sentimientos, puede hacer más eficaces sus encuentros clínicos.

Ruiz Moral (2003), citado por Loriente (2009), agrega a los modelos consignados el tipo de relación médico - paciente "consumista", caracterizado por enfermos más jóvenes, con un nivel educativo mayor y que son más demandantes y asertivos, que ejercen un mayor control sobre el médico. 
En cuanto a si existe un tipo de relación mejor que otro, Ruiz Moral (2003) plantea que no puede afirmarse de forma categórica, pero sí otorga un lugar de importancia a las habilidades comunicacionales del médico para adoptar un tipo de relación acorde a las necesidades del paciente, que puede tener matices en sus diferentes etapas, y tiene en cuenta sus aspectos psicológicos y sociales. En la literatura científica, diversos estudios aportan evidencia empírica en este sentido (D'Anello, 2006; Prados Castillejo et al., 2000). Se destaca la escucha activa del médico para el logro de una comunicación más eficaz con sus pacientes, así como la participación activa del paciente que contribuye a la comprensión de la información y a incrementar su satisfacción y confianza en el médico, resultando positivo para el cumplimiento y la efectividad de los tratamientos (Balint, 1961; Mira y Rodríguez Marín, 2001).

La idea del paciente competente, señala Mira (2005), se vincula con el concepto de autoeficacia, desarrollado tempranamente en 1977 como una alternativa al paternalismo médico. Este tema fue poco tratado y en las últimas décadas aumenta su interés. También existen talleres de capacitación al paciente en el manejo de enfermedades crónicas. La educación para la salud viene demostrando la eficacia de diferentes formatos y tipos de educación en el tratamiento antihipertensivo y la adherencia (Galarza et al., 2013; Danet et al., 2012).

\subsection{Habilidades comunicacionales del médico en la entrevista clínica}

\section{a) Definición}

Canovaca Vega (2010) las define como aquellas conductas e instrumentos técnicos utilizados por el médico en la entrevista, para la ejecución de tareas. Incluye principalmente técnicas de apoyo narrativo (contacto visual facial, facilitaciones, señalamientos); técnicas de información (ejemplificación, información por escrito, racionalización del tratamiento, complemento visual táctil); y técnicas de negociación (respuesta evaluativa, paréntesis, doble pacto, cesión intencional).

Moore y Gómez del Río (2007) afirman que el éxito de cualquier entrevista clínica depende de la calidad de la comunicación médico - paciente. Con el uso de habilidades de comunicación efectiva se busca aumentar la precisión diagnóstica, la eficiencia en términos de adherencia al tratamiento, y construir un apoyo para el paciente, según afirman Razavi (2000) y Stewart (1996), citados por Vidal y Benito (2010).

Asimismo, Moore y colaboradores (2010) plantean que la comunicación efectiva se caracteriza por asegurar una interacción, en vez de una transmisión directa o simple entrega de información. Reduce la incertidumbre innecesaria, requiere planificación, demuestra dinamismo y sigue un modelo helicoidal (comunicar es construir sentido en la interacción) más que lineal (comunicar es transmitir y descifrar una información desde un determinado código).

La importancia de las habilidades comunicacionales, según aducen Torío y García (1997), radica en que se relaciona con aspectos tan variados como la satisfacción, el cumplimiento, la percepción de la competencia profesional, la frecuencia de problemas 
legales en relación a la mala praxis e incluso el pronóstico de la enfermedad o el estado de salud general del enfermo.

b) Tiempo de la entrevista en la comunicación médico - paciente.

La duración de las consultas en atención primaria se relaciona con aspectos de la comunicación médico - paciente y, de forma indirecta, con el estilo de la entrevista médica. El sistema de salud impone tiempos de atención cada vez más breves. Está demostrado, según plantea Bellón (2001), que la menor duración de las consultas se relaciona con una mayor prescripción de medicación sintomática; mayor número de derivaciones; menor posibilidad de que el paciente haga preguntas o exprese sus opiniones; menor información por parte del médico de diagnósticos y tratamientos; menos consejos preventivos; peor satisfacción del paciente y del médico; y un aumento de la repetición de visitas de los enfermos. Según este autor, en general el abordaje psicosocial requiere de 3 a 6 minutos más de consulta.

Por su parte, Moore (2010) y Loriente (2009) señalan que una vez que las habilidades comunicacionales son manejadas adecuadamente, lo fundamental es la calidad de la relación que se establece durante el tiempo que se permanece en consulta.

c) Habilidades específicas que marcan la diferencia en la comunicación médico - paciente.

De la Rosa Legón (2010) y van der Hofstadt (2004) sostienen que para lograr el éxito en la persuasión de los pacientes el equipo de salud debe tener conocimientos científicos sólidos y el profesional debe tener conciencia de su competencia comunicativa, lo que incluye la esfera afectiva motivacional (motivos, propósitos, expectativas y vivencias de ambos actores sociales), donde la subjetividad de los profesionales y los pacientes es un elemento esencial. Teniendo en cuenta esto, Alonso y Fuentes (2008) proponen que para lograr un buen clima comunicacional es necesario ofrecer un adecuado marco de confidencialidad, brindar tiempo y espacio, voz suave, pausada, mirada sincera, escucha activa, silencios terapéuticos y gestos reafirmantes del espacio. Se logra así menor tensión y ansiedad, y mayor exposición de contenidos.

\subsection{Satisfacción del paciente}

\section{a) Definición}

Según Carr y Hill (1992) la satisfacción del paciente se basa en la diferencia entre sus expectativas y la percepción de los servicios que ha recibido. De esta manera, las percepciones subjetivas, acompañadas de las expectativas previas de los usuarios, configuran la expresión de la calidad del servicio. En el ámbito de la salud se identifica la satisfacción del paciente con la cantidad y calidad de la información que reciben, basándose en la comunicación del profesional de la salud con el enfermo como el criterio de satisfacción.

Por su parte, a partir del paradigma de la disconformidad (Linder-Pelz, 1982), se define la satisfacción como un concepto multidimensional cuyos componentes varían 
en función del tipo de prestación de que se trate (hospital de agudos, consulta de enfermos crónicos, urgencias, etc.), más directamente relacionada con el componente afectivo de la actitud hacia el sistema sanitario o cualquiera de sus unidades (consultas o profesionales) y que puede explicarse por la no confirmación en las expectativas, donde la diferencia entre expectativas y percepciones es el elemento clave.

\section{b) Aspectos más valorados por los pacientes y nivel de satisfacción}

Diversos estudios mostraron que los aspectos centrales de un servicio de Atención Primaria y que generan un mayor nivel de satisfacción en los pacientes son aquellos que se refieren a la relación establecida con el médico. Hallaron, además, que conviene mejorar las instalaciones y el equipamiento de los centros de salud, unido a la accesibilidad al servicio; y que el estilo de comunicación centrado en el paciente se ha asociado positivamente con la satisfacción y otros resultados de la consulta (Varela Mallou, 2003; Ruiz Moral et al., 2011).

Por último, señalan que la satisfacción del paciente con los servicios sanitarios es un concepto complejo, que está relacionado con una gran variedad de factores: el estilo de vida; las experiencias previas; el tiempo dedicado a la consulta; la calidad científicotécnica del profesional; la empatía percibida por el paciente; las expectativas de futuro y los valores del individuo y de la sociedad (Martín Alfonso, 2009; De los Ríos y Ávila Rojas, 2004; Mira, 1998).

\subsection{Adherencia al tratamiento médico}

a) Definición

El proyecto "Adherencia a los tratamientos a largo plazo: Pruebas para la acción", de la Organización Mundial de la Salud (Sabaté, 2004), considera a la adherencia terapéutica como el grado en que el comportamiento de una persona, relativo a tomar medicamentos, seguir un régimen alimentario o ejecutar cambios en el estilo de vida, se corresponde con las recomendaciones acordadas con un prestador de asistencia sanitaria. Diversos autores coinciden en definir este concepto como un proceso en el que los pacientes participan de modo activo y se produce una relación de colaboración con los profesionales, con el fin de obtener un resultado adecuado. Así concebida, la adherencia supera el mero cumplimiento obediente y pasivo, que fomentaría poco la responsabilidad del individuo para el control de su enfermedad (Alfonso, Bayarre y Grau, 2008; Ingaramo et al., 2005). Según plantea Varela (2010), su importancia está dada por las repercusiones que tiene desde el punto de vista clínico, económico y psicosocial; además de su efecto en la calidad de la atención y en la relación médicopaciente (Di Matteo, 2002).

Según Ortego Maté (2011) el incumplimiento o la deficiente adherencia a las prescripciones de salud se asocia a una serie de consecuencias negativas, las cuales se pueden agrupar en cuatro grandes apartados:

- Incremento de los riesgos, la morbilidad y la mortalidad,

- Aumento de la probabilidad de cometer errores en el diagnóstico y tratamiento, 
- Crecimiento del coste sanitario.

- Desarrollo de sentimientos de insatisfacción y problemas en la relación que se establece entre el profesional y el paciente.

Todo ello hace necesario que los profesionales y las instituciones de la salud adopten estrategias destinadas a prevenir o disminuir la falta de adherencia.

b) Factores que inciden en la adherencia terapéutica

En cuanto a las causas de falta de adherencia al tratamiento, distintos autores, coinciden en que son múltiples los factores que la determinan. Se pueden agrupar en dos grandes grupos:

- Variables derivadas del médico, del paciente, del tipo de relación médico-paciente que se establezca;

- Variables derivadas de la salud (tipo de enfermedad y gravedad), de la complejidad del tratamiento, de aspectos familiares, sociales, organizacionales y económicos.

En este último aspecto, poseer algún tipo de cobertura u obra social determina una mejor adherencia, lo que puede ser interpretado como un indicador más, de las dificultades socioeconómicas que padece un grupo de la población (Ingaramo, Vita y Bendersky, 2005).

En lo que respecta a las variables organizacionales, la frecuencia en las citas, la distancia respecto a los recursos asistenciales, la falta de médico en el momento que el paciente lo requiere, o los cambios de médicos, el transporte, pueden ser dificultades añadidas para la adherencia al tratamiento. Se observó también un mayor riesgo de abandono en sistemas con una atención centralizada, donde se evidenciaron dificultades ligadas a la organización de los hospitales de mayor complejidad: alta demanda, poco personal, largas esperas y atención menos personalizada, entre otros aspectos (Alfonso, Sairo y Bayarre, 2003; Ortiz y Ortiz, 2007; Ginarte Arias, 2001; Ingaramo, Vita y Bendersky, 2005; Alfonso, 2006; Arrossi et al., 2007).

Finalmente, se demostró que la satisfacción y la comunicación del paciente con el equipo de atención en salud son variables que pueden contribuir a la adherencia al tratamiento en pacientes con enfermedades crónicas (D’Anello et al.2009; Goldring et al., 2002; Di Matteo et al.,1993; Di Matteo et al., 2000).

\section{Conclusiones}

A partir del análisis de la bibliografía seleccionada, se concluye que:

- La importancia de la comunicación en la relación médico-paciente radica en la correlación entre la calidad del proceso comunicativo, la satisfacción del usuario y el cumplimiento de los tratamientos. 
- No hay un tipo de relación médico - paciente mejor que otra. Las habilidades comunicacionales médicas cobran importancia para, al tener en cuenta los aspectos psicosociales de los pacientes, adaptarse a sus necesidades, a las diferentes etapas del tratamiento y lograr mayor eficacia.

- Se destacan la escucha activa del profesional y el logro de la participación activa del paciente como habilidades importantes para el cumplimento y efectividad de los tratamientos.

- La comunicación efectiva se caracteriza por asegurar una interacción, en vez de una transmisión directa o simple entrega de información.

- De acuerdo con la literatura analizada, la idea del paciente competente ha sido poco estudiada. En concreto, de qué manera puede mejorarse la comunicación con el médico, lograr un mayor compromiso con su tratamiento, una mayor adherencia a partir del entrenamiento a pacientes sobre cómo manejarse en las entrevistas con el profesional. Se trata de un tema importante a desarrollar por futuras investigaciones.

- Hay posiciones diversas sobre el tiempo adecuado de consulta. Bellón (2001) considera que, en general, el abordaje psicosocial requiere de 3 a 6 minutos más de consulta, en línea con autores como Sogi y colaboradores (2006) y Barca y colaboradores (2004) que plantean la importancia de mayor tiempo de consulta para una comunicación eficaz. En cambio, autores como Moore y colaboradores (2010) y Loriente y colaboradores (2009) señalan que una vez que las habilidades comunicacionales son manejadas adecuadamente no demandan mucho más tiempo que el habitual; para estos autores, lo trascendente es la calidad de la relación que se establece durante el tiempo que se permanece en consulta.

- Hay consenso en la literatura científica en que la interacción médico - paciente, la satisfacción del enfermo y la adherencia al tratamiento son fenómenos multidimensionales, en los que inciden cuestiones del médico, del paciente, del sistema de salud y del tipo de enfermedad, entre otras variables.

- En cuanto a satisfacción, la controversia estuvo dada en principio sobre la definición del concepto y los instrumentos para medirla. Otro punto refiere a qué factores del servicio de salud resultan más importantes para que los pacientes se sientan satisfechos. En un principio la satisfacción estaba ligada a la calidad asistencial, al rendimiento del servicio que era evaluado por los usuarios. Luego la satisfacción del paciente pasó a ser, ante todo, una medida del resultado de la interacción entre el profesional de la salud y el paciente. También se empezó a considerar su carácter multifactorial.

- En cuanto a la adherencia, las controversias estuvieron ligadas, en un comienzo, a su definición conceptual. El concepto de adherencia resulta más adecuado en comparación con el de cumplimiento (compliance) para abordar un fenómeno complejo y multidimensional como es la interacción entre médico - paciente, porque describe 
un estilo de comunicación más participativo, donde ambos actores sociales deciden en conjunto los pasos a seguir en el tratamiento de la enfermedad.

- Las principales críticas a la medición de la adherencia apuntan a las técnicas que evalúan exclusivamente el cumplimiento del tratamiento a nivel medicamentoso, sin tomar en cuenta aquellas indicaciones que se refieren a cambios de hábitos o estilos de vida.

- Se halló un mayor riesgo de abandono al tratamiento en sistemas de salud con una atención centralizada, alta demanda, poco personal, largas esperas y atención menos personalizada.

- Altos niveles de adherencia se asociaron con modelos de comunicación que consideran las actitudes, las creencias y las preferencias de los pacientes, y permiten un proceso de negociación con mayor acuerdo y comprensión.

- La satisfacción y la comunicación del paciente con el equipo de atención en salud, son variables que pueden contribuir a la adherencia al tratamiento en pacientes crónicos.

- Los factores que más influyen en los pacientes para adherir al tratamiento son: la relación que se establece con el médico (interacción, comunicación); las cuestiones institucionales (obstáculos de la organización de salud).

- En cuanto a las investigaciones realizadas sobre la relación médico - paciente, Martínez y Leal (2003) plantean que el problema de las mismas es que en el intento de dar con el "cómo hacerlo" parecen haber perdido el rumbo hacia el "qué hay que hacer". Es decir, los trabajos se ocupan más de "la forma" que de "el fondo".

- En el ámbito científico, se plantea la necesidad de continuar con el diseño y desarrollo de investigaciones para identificar aquellos factores que muestran una influencia decisiva en la relación médico-paciente: el contexto sanitario; los factores personales; los factores comunicacionales; el valor de la relación continuada en el tiempo con los pacientes; el papel que los pacientes dan a sus familias; y las peculiaridades culturales y expectativas diferenciales de los pacientes frente al médico (Ruiz, 2003).

\section{Referencias}

Alonso, S; Fuentes, J. et al. (2008). Como interpretar la comunicación no verbal. Actualizaciones. Formación Médica Continuada en Atención Primaria. Vol. 15, n 5, pp. 275-81.

Arrossi, S.; Matos, E.; Zengarini, N.; Roth, B.; Sankaranarayanan, R. y Parkin, M. (2007). The socio-economic impact of cervical cancer on patients and their families in Argentina, and its influence on radiotherapy compliance. Results from a cross-sectional study. Gynaecologic Oncology. Vol. 105, n² 2, pp. 335-340. 
Balint, M. (1961). El médico, el paciente y la enfermedad. Buenos Aires: Editorial Ateneo.

Barca, I.; Parejo, R.; Gutiérrez, P; Fernández, F; Alejandre, G y López, F. (2004). La información al paciente y su participación en la toma de decisiones clínicas. Atención Primaria. Vol. 33, $\mathrm{n}^{\circ} 7$, pp. 361-7.

Bellón Saameño, J.A. y Martínez Cañabate, T. (2001). La investigación en comunicación y salud. Una perspectiva nacional e internacional desde el análisis bibliométrico. Atención Primaria. Vol. 27, $\mathrm{n}^{\circ}$ 7, pp. 452-58.

Bolaños, E. y Sarriá Santamera, A. (2003). Perspectiva de los pacientes sobre la diabetes tipo 2 y relación con los profesionales sanitarios: un estudio cualitativo. Atención Primaria. Vol. 32, n 4, pp. 195-201.

Canovaca Vega, AM. (2010). Perfil Comunicacional del Médico de Atención Primaria en las consultas de demanda: Validación de un cuestionario. Universidad de Córdoba. Facultad de Medicina. Departamento de Medicina. Córdoba: Servicio de Publicaciones de la Universidad de Córdoba.

D'Anello Koch S. (2006). Cumplimiento del tratamiento médico: Aspectos psicosociales. Cap. 10. En Esqueda Torres L., Escalante L. G, Barreat Montero Y., D' Orazio A. K., Escalante K. L., Ángeles F. y Linares F. Aportes a la Psicología Social de la Salud. Mérida (Venezuela): Ediciones Vicerrectorado Académico de la Universidad de Los Andes.

D’Anello Koch S.; Barreat, Y.; Escalante, G.; D’Orazio, A. K. y Benítez, A. (2009). Relación médico paciente y adherencia a tratamiento. Revista MédULA. Vol. 18, $\mathrm{n}^{\circ} 1 ., \mathrm{pp}$. 33-39.

Danet, A; Escudero, M. J.; Prieto, M. A., March Cerda y Carles, J. (2012). Comunicación paciente-médico y toma de decisiones en salud en Andalucía. Expectativas de pacientes crónicos del SSPA. Revista de Comunicación y Salud. Vol. 2, nº 2, pp. 63-77.

De la Rosa Legón, M.; Vega González, N. y Brito Gómez, L. (2010). El paradigma médico social y la competencia comunicativa del profesional de ciencias médicas. Revista Habanera de Ciencias Médicas. Vol. 9, n³, pp. 433-40.

De los Ríos Castillo. J.L. y Ávila Rojas. T.L (2004). Algunas consideraciones en el análisis del concepto: satisfacción del paciente. Investigación y Educación en Enfermería. Vol. XXII, $\mathrm{n}^{\circ} 2$.

Di Matteo, M.R. (2000). Practitioner-family-patient communication in pediatric adherence: Implications for research and clinical practice. En D. Drota, (Ed.). Promoting adherence to medical treatment in childhood chronic illness: Concepts, methods, and interventions (pp. 237-258). New York: Erlbaum.

Di Matteo, M.R.; Sherbourne, C.D.; Hays, R.D. et al. (1993). Physicians characteristics influence patient adherence to medical treatment: Results from the medical outcome study. Health Psychology. Vol. 12, n² 2, pp. 93-102. 
Di Matteo, M. R.; Giordani, P.; Lepper, H. y Croghan, T. (2002). Patient adherence and medical treatment outcomes: a meta-analysis. Medical Care. Vol. 40, nº 9, pp. 794-811.

Dörr Anneliese A. (2004). Acerca de la comunicación médico-paciente desde una perspectiva histórica y antropológica. Revista Médica de Chile. Vol. 132, n¹1, pp. 1431-36.

Froján M. y Rubio R. (2005). Discriminant anlysis of tretment adherente in insulindependent diabetes mellitus. Psychology in Spain. Vol 9, pp. 41-48.

Galarza, C.; Cuffaro, P.; Rada, M. y Janson, J. (2013). La educación y el tratamiento del paciente hipertenso. En Llambí, G. H.y Piskorz, D. Hipertensión arterial, epidemiología, fisiología, fisiopatología, diagnóstico y terapéutica (pp. 479-484). Buenos Aires: Intermédica.

Ginarte Arias Y. (2001). La adherencia terapéutica. Revista Cubana de Medicina General Integral. Vol. 17, $\mathrm{n}^{\circ}$ 5, pp. 502 - 505.

Goldring A.; Taylor S, Kemeny M. et al. (2002). Impact of health beliefs, quality of life, and the physician - patient relationship on the treatment intentions of inflammatory bowel disease patients. Health Psychology. Vol. 21, pp. 218-228.

Hernández-Torres I. et al. (2006). Importancia de la comunicación médico paciente en medicina familiar. Medigraphic Artemisa. Vol. 8, n² 2, pp. 137-143.

Ingaramo, R.; Vita, N. y Bendersky M. (2005). Estudio Nacional sobre Adherencia al Tratamiento. Revista de la Federación Argentina de Cardiología. Vol 34, pp. 104-11.

Laine, C. y Davidoff F. (1996). Patient-centered medicine. A professional evolution. Journal of the American Medical Association. Vol. 275, pp. 152-6.

Lassen L. C. (1991). Connections between the quality of consultations and patient compliance in general practice. Family Practice. Vol. 8, n² 2, pp. 154-160.

Ley P. (1983). Patients understanding and recall in clinical communication failure. En: Pendleton D, Hasler J (eds.). Doctor-patient communication (pp. 89-107). Londres: Academic Press.

Linder-Pelz S. (1982). Social psychological determinants of patient satisfaction. Social Science and Medicine. Vol. 16, $n^{\circ} 5$, pp. 583-9.

Loriente Arín, N. y Serrano del Rosal, R. (2009). Hable con los pacientes, no para ellos. Revista Internacional de Sociología. Vol. 67, n² 2, pp. 309-28.

Martín Alfonso, L.; Sairo, M. y Bayarre Vea H. (2003). Frecuencia de cumplimiento del tratamiento médico en pacientes hipertensos. Revista Cubana de Medicina General Integral. Vol. 19, n 2. 
Martín Alfonso, L. M. (2006). Repercusiones para la salud pública de la adherencia terapéutica deficiente. Revista Cubana de Salud Pública. Vol. 32, n³, Julio-Septiembre.

Martín Alfonso, L.; Bayarre Vea, H. y Grau Ábalo, J.A. (2008). Validación del cuestionario MBG (Martín-Bayarre-Grau) para evaluar la adherencia terapéutica en hipertensión arterial. Revista Cubana de Salud Pública. Vol. 34, n¹, Enero-Marzo.

Martín Alfonso L. (2009). Adherencia al tratamiento en hipertensos de áreas de salud del nivel primario. Tesis Doctoral en Ciencias de la Salud. Ministerio de Salud Pública. Escuela Nacional de Salud Pública. Revista Cubana de Salud Pública [versión electrónica]. Disponible en http://www.sld.cu/galerias/pdf/sitios/revsalud/tesislibertad.pdf. Recuperado el 21 de octubre de 2012.

Martínez S. y Leal F. (2003). La calidad clínica de la atención y el problema de la relación médico-paciente. Anales Médicos Asociación Médica del American British Cowdray. Vol. 48, n 4, pp. 242-254.

McLane, C.; Zyzanski, S. y Flocke S. (1995). Factors associated with medication noncompliance in rural elderly hypertensive patients. American Journal of Hypertension. Vol. 8, n² 2, pp. 206-209.

Mira, J.; Lorenzo, S.; Rodríguez-Marín J.; Aranaz, J. y Sitges E. (1998). La aplicación del modelo europeo de gestión de la calidad total al sector sanitario: ventajas y limitaciones. Revista Calidad Asistencial. Vol. 13, pp. 92-7.

Mira, J. y Rodríguez-Marín, J. (2001). Análisis de las condiciones en las que los pacientes toman decisiones responsables. Medicina Clínica. Vol. 116, pp. 104-110.

Mira, J. (2005). El paciente competente, una alternativa al paternalismo. Monografías Humanitas $N^{0}$ 8. Fundación Medicina y Humanidades. Disponible en http://www.fundacionmhm.org/pdf/Mono8/Articulos/articulo8.pdf. Recuperado el 17 de agosto de 2012.

Moore, P y Gómez del Río, G. (2007). Comunicarse Efectivamente en Medicina. ¿Cómo adquirir habilidades comunicacionales durante la carrera de medicina? Ars Médica, Revista de Estudios Médico Humanísticos. Vol. 15, n 15, pp. 171-81.

Moore, P.; Gómez, G.; Kurtz, S. y Vargas, A. (2010). La comunicación médico - paciente: ¿Cuáles son las habilidades efectivas? Revista Médica de Chile. Vol. 138, pp. 1047-54.

Ortego Maté M. C. (2011). Adherencia al tratamiento antirretroviral. Tesis Doctorales en Red (TDR). Editorial Universidad de Cantabria. Disponible en http://hdl.handle.net/10803/51516. Recuperado el 11 de marzo de 2012.

Ortiz, M., Ortiz, E. (2007). Psicología de la salud: Una clave para comprender el fenómeno de la adherencia terapéutica. Revista Médica de Chile. Vol. 135, pp. 647-52. 
Pérez Cicili A., Vidaillet Calvo E. C., Carnot Pereira J. y Duane Machado O. J. (2003). La relación médico-paciente en el Sistema Nacional de Salud. Revista Cubana Medicina General Integral [versión electrónica]. Disponible en: http://scielo.sld.cu/scielo. php?script=sci_arttext\&pid=S0864-21252003000600006\&Ing=es. Recuperado el 26 de febrero de 2012.

Pérgola F. M. (2002). Cultura, Globalización y Medicina. Buenos Aires: Editorial El Guión.

Pérgola F. M. (2010). La relación entre el médico y su paciente en la era electrónica. Anales de la Academia Nacional de Ciencias de Buenos Aires [versión electrónica]. Disponible en http://www.ciencias.org.ar/user/FILE/P\%C3\%A9rgolainc.pdf. Recuperado el 27 de septiembre de 2012.

Piñeiro, D. J. (2005). Pacientes o clientes, médicos o prestadores. Revista Argentina de Cardiología. Vol.73, n 5, pp. 402-3.

Prados Castillejo, J.A; Cebria Andreu, J: y Bosch Fontcuberta J.M. (2000). Comunicación centrada en el paciente: una aplicación clínica. Formación Médica Continua en Atención Primaria. Vol. 7, pp. 83 -95.

Razavi, D.; Delvaux, N.; Marchal, S.; De Cock, M. et al. (2000). Testing health care professionals' communication skills: the usefulness of highly emotional standardized role-playing sessions with simulators. Psycho-Oncology. Vol. 9, n² 4, pp. 293-302.

Robbins, J.; Bertakis, K; Helms, L; Azari, R. et al. (1993). The influence of physician practice behaviors on patient satisfaction. Family medicine. Vol. 25, nº 1, pp. 17-20.

Rodríguez Silva, H. (2006). La relación médico-paciente. Revista Cubana de Salud Pública. Vol. 32, nº 4, Diciembre.

Ruiz Moral, R.; Rodríguez J. y Epstein, R. (2003). ¿Qué estilo de consulta debería emplear con mis pacientes?: reflexiones prácticas sobre la relación médico-paciente. Atención Primaria. Vol. 32, nº 10, pp. 594-602.

Ruiz Moral, R.; Pérula de Torres, L.A.; Muñoz, M. et al. (2011). Satisfacción de los pacientes con la comunicación en las consultas de medicina de familia: una comparación de métodos para investigar necesidades no satisfechas. Revista Española de Salud Pública. Vol. 85, n³ 3 pp. 317-24.

Sabaté E. (2004). Adherencia a los tratamientos a largo plazo: pruebas para la acción. Organización Mundial de la salud. Disponible en: http://www.paho.org/spanish/AD/ DPC/NC/adherencia-largo-plazo.pdf.Recuperado el 13 de febrero 2012.

Sogi C., Zavala S., Oliveros M. y Salcedo C. (2006). Autoevaluación de formación en habilidades de entrevista, relación médico - paciente y comunicación en médicos graduados. Anales Facultad de Medicina de Perú. Disponible en: http://www.scielo.org. pe/pdf/afm/v67n1/a06v67n1. Recuperado el 18 de noviembre de 2012. 
Starfield, B.; Wray, C.; Hess, K. et al. (1981). The influence of patient - practitiones agreement on outcome of care. American Journal of Public Health. Vol. 71, $\mathrm{n}^{\circ} 2$, pp. 127-31.

Stewart M.A. (1996). Effective physician-patient communication and health outcomes: a review. Canadian Medical Association Journal. Vol. 152, pp. 1423-33.

Torío, J. y García, M.C. (1997a). Valoración de la orientación al paciente en las consultas médicas de atención primaria. Atención Primaria. Vol. 20, pp. 17-24.

Torío, J. y García, M.C. (1997b). Relación médico-paciente y entrevista clínica (II): opinión y preferencias de los médicos. Atención Primaria. Vol. 19, pp. 27-34.

Torío, J. y García, M.C. (1997c). Relación médico-paciente y entrevista clínica (I): opinión y preferencias de los usuarios. Atención Primaria. Vol. 19, pp. 18-26.

Van-der Hofstadt Román C. J. y Navarro Cremades F. (2004). Las habilidades de comunicación como elemento básico en la formación del médico. SEMERGEN - Medicina de Familia. Vol. 30, nº 11, pp. 552-556.

Varela, M.T. (2010). Evaluar la adherencia al tratamiento en la HTA. Pensamiento Psicológico. Vol. 7, no 14, pp. 127-140.

Varela Mallou, J.; Rial Boubeta, A. y García Cueto, E. (2003). Presentación de una escala de satisfacción con los servicios sanitarios de atención primaria. Psicothema. Vol. $15, n^{\circ} 4$, pp. 656-61.

Vidal y Benito M. C. (2010). La Relación Médico Paciente. Bases para una comunicación a medida. Buenos Aires: Lugar Editorial. 